\title{
Mentorando meninas iniciantes em programação: um estudo de caso
}

\author{
Fabrizio Honda Franzoia ${ }^{1}$, Fernanda G. de Sousa Pires ${ }^{1}$, Marcela Sávia P. Pessoa ${ }^{1}$ \\ ${ }^{1}$ Laboratório de Tecnologias Educacionais - Universidade do Estado do Amazonas \\ Manaus, AM - Brazil \\ \{fhf.lic17, fpires, msppessoa\}@uea.edu.br
}

\begin{abstract}
This article discusses models, methodologies, biases and their impacts for a group of freshmen girls on a computer course. As well, discussed about the creation of focus groups, for girls with a view to empowerment as an initial, empowering measure. The method used was the case study with a quality-quantity approach. Results show that the measure: "creation of a group of mentored studies" was a good initiative given the degree of acceptance and results evaluated qualitatively (students' description) and quantitative (evaluation of contests).

Resumo. O presente artigo discute modelos, metodologias, preconceitos e os seus impactos para um grupo de meninas calouras em um curso de computação. Bem como levanta altercações em torno da criação de grupos de estudos focais para meninas com vistas ao empoderamento como medida inicial fortalecedora. O método utilizado foi o estudo de caso com uma abordagem do tipo quali-quanti. Resultados apontam que a medida: "criação de grupo de estudos mentorados" se mostrou uma boa iniciativa dado o grau de aceitação e de resultados avaliados de forma qualitativa (descrição das alunas) e quantitativa (avaliação de contests).
\end{abstract}

\section{Introdução}

Historicamente a área de Computação foi impulsionada por mulheres, desde o primeiro algoritmo, por Ada Lovelace, até Carol Shaw, a primeira desenvolvedora de jogos de computadores. Porém, ao longo dos anos é perceptível a diminuição da participação feminina tanto nos cursos superiores relacionados à área de Computação (Pessoa et al., 2017), quanto no mercado de trabalho. Em 2010, aproximadamente $\quad 520.000$ profissionais atuavam em atividades relacionadas à tecnologia da informação, desse total, apenas $25 \%$ eram mulheres (IBGE, 2010).

Em se tratando de Computação é imprescindível citar a Programação, uma área promissora para o crescimento pessoal/profissional (Santos et al., 2018) e que instiga não só conhecimentos tecnológicos, mas também o desenvolvimento de habilidades para a resolução de problemas, como o Pensamento Computacional (Wing, 2006). Bosse (2015) realizou um levantamento em uma universidade para investigar a afirmação de que a disciplinas introdutórias de programação mantém altos índices de reprovação. Dados apontaram que a taxa de reprovação varia entre 30 a $50 \%$ e, dentre os aprovados, $25 \%$ cursaram a disciplina uma ou mais vezes.

Considerando os altos índices de reprovação em disciplinas iniciais de programação e a menor porcentagem de mulheres nos cursos, este artigo investiga: i) fatores que podem ter culminado em reprovação de mulheres na disciplina de Introdução à Programação e ii) estes fatores podem ser minimizados através da 
participação em Grupo de Estudos em Programação. Com base nestes questionamentos, a proposta do artigo é apresentar um estudo de caso realizado em universidade pública.

O artigo está organizado como segue: A Seção 2 contém o Referencial Teórico e Trabalhos Relacionados, na Seção 3 é descrita a metodologia desenvolvida no Grupo de Estudos em Programação, técnica de pesquisa e método de análise, na Seção 4 são apresentadas algumas observações iniciais e os resultados e na Seção 5 são registradas as considerações finais e trabalhos futuros.

\section{Referencial Teórico e Trabalhos Relacionados}

A partir da análise de trabalhos nacionais da Literatura, observou-se que o cenário brasileiro em cursos de Computação, mais especificamente em disciplinas de Programação, apresenta uma série de desafios e dificuldades: na compreensão dos conteúdos em sala de aula (Giraffa \& da costa Mora, 2013), na ausência de interesse em procurar entender os exercícios, a falta de estratégias para resolução de problemas, índices significativos de reprovação (Bosse \& Gerosa, 2015), dentre outros.

A Programação é de suma importância para o graduando em Computação; o entendimento de Lógica de Programação e a Aprendizagem de Algoritmos são pilares essenciais para sua compreensão. Contudo, a aprendizagem de Algoritmos é uma atividade desafiadora, principalmente pela dificuldade de abstrair o conteúdo e em reconhecer procedimentos importantes para alcançar a solução ou reprovação (Castro et al., 2009). Em parte dos estudantes, a reprovação tem cargas negativas e, consequentemente, agravando desinteresses e diminuição na autoestima (Deters et al., 2008).

Além dessas dificuldades, tópicos mais específicos rodeiam as mulheres : falta de identificação com o curso por não terem conhecimento de mulheres destaques na área, inibição por serem minoria, ocasionando ausência de vontade para tirar dúvidas em sala de aula. Nesse contexto, surgem programas focados no incentivo de mulheres em áreas de Computação, como o Cunhantã Digital - envolvem ações e projetos através de palestras e atividades lúdicas em eventos científicos (Lauschner et al., 2016) -, Meninas Digitais - coordenado pela Sociedade Brasileira de Computação (SBC), com enfoque maior em mulheres nos anos finais dos Ensinos Fundamental e Médio (Maciel \& Bim, 2017) - GRACE - Grupo de Alunas nas Ciências Exatas que objetiva desenvolver atividades de extensão em áreas de ciência em tecnologia, com um público feminino, mais especificamente, da cidade de São Carlos.

Trabalhos como o de Sousa et. al (2018) analisam desempenho e participação feminina em um clube de programação com estudantes de ensino médio em escola pública, através de metodologia baseada em projetos e ancorada a teoria construcionista de Seymour Papert. Resultados alcançados possibilitaram às meninas conhecer a história da computação, sua importância e a participação das meninas na criação do Clube de Programação. Já (Alves et al., 2017) descrevem um relato da iniciativa do uso da técnica Dojo de Programação, objetivando a capacitação e incentivo ao público feminino de cursos de Computação. Com o andamento das três etapas em que o trabalho foi dividido, as autoras comentam que, as estudantes aprovaram a iniciativa, aprendendo participativa e colaborativamente, superando limitações. 


\section{Metodologia aplicada ao Grupo de Estudos em Programação}

O Grupo de Estudos em Programação foi formado com o intuito de ministrar um curso de Introdução à Programação com a linguagem Python para estudantes do sexo feminino de cursos superiores em Computação. Todas as alunas haviam reprovado na primeira disciplina de programação da matriz curricular. O curso contou com a participação de 6 monitores responsáveis por fazer o planejamento das aulas e ministrálas, de acordo com o conteúdo. A metodologia de trabalho seguiu as etapas descritas nesta Seção.

\subsection{Pré-teste e Andamento do curso}

Aplicou-se um pré-teste para analisar o nível de conhecimento que as estudantes tinham em lógica de programação e em Python, abordando questões de entrada e saída de dados, operações aritméticas básicas, estruturas condicionais e laços de repetição. As aulas iniciaram-se logo em seguida, conforme o conteúdo programático e planejamento estabelecidos pelos monitores. Para elaboração da sequência de cada aula e seus respectivos conteúdos, utilizou-se como base o Plano de Ensino de disciplinas de Introdução à Programação. As aulas prosseguiram com esta sequência: introdução à lógica de programação e Python, operadores e estruturas condicionais, laços de repetição, listas (vetores), funções e listas de listas (matrizes).

O curso estendeu-se por um mês, totalizando 60 aulas: $50 \%$ de aulas expositivas com conteúdo, exemplos e exercícios relacionados e 50\% aulas de aplicação de questões de competições (aqui chamadas de contests). Além das aulas, houve momentos de conversação entre os monitores e estudantes, em busca de feedback sobre o andamento do curso. A Figura 1 ilustra momentos do curso.

\subsection{Os contests}

Os contests foram aplicados após determinado conteúdo ser ministrado e continham questões relacionadas a este. A quantidade de questões dos contests variava entre $6 \mathrm{e}$ 10, todas retiradas da plataforma de juiz online, URI Online Judge. As estudantes acompanhavam, em tempo real, a classificação do contest (posição, questões tentadas e resolvidas, tempo de resolução), através do A2 Online Judge, plataforma de juiz online.

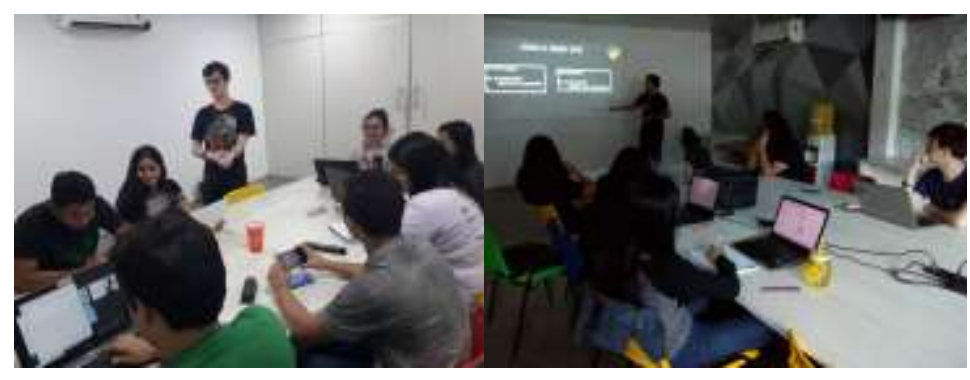

Figura 1. Registros das aulas expositivas

\subsection{Técnica de Pesquisa e Métodos de análise}

Como técnica de pesquisa, foi utilizado um estudo de caso, estratégia de pesquisa onde está contido um método abrangente para coletas e análise de dados (Yin, 2015). Em relação à avaliação do Grupo de Estudos, foi utilizada uma abordagem quali-quanti, considerando as respostas das meninas por meio de um questionário (qualitativa) e o nível de evolução na resolução dos contests (quantitativa). 


\section{Observações iniciais e Resultados}

Observando o fluxo das primeiras aulas, percebeu-se certas particularidades das estudantes: $30 \%$ tinham um conhecimento mais apurado em lógica de programação, decorrente do contato prévio com a linguagem $\mathrm{C} ; 35 \%$ possuíam um domínio maior, em relação as demais, na sintaxe de Python e; 35\% apresentaram dificuldades iniciais no entendimento do conteúdo, consequentes da ausência de concentração e presença nas aulas. O curso foi prosseguindo e as estudantes demonstraram maior nível de engajamento nas aulas, dúvidas perguntadas com mais frequência aos monitores, resolução de questões entre si, estudos em programação dentro e fora da universidade, etc. É importante salientar também que, durante os contests, as estudantes estavam solucionando subproblemas das questões com maior facilidade; estes estavam presentes, em grande parte, no pré-teste.

Os resultados foram satisfatórios. Mais de $75 \%$ das alunas obtiveram nota superior a 7,0. Porém, em 25\% dos casos, a média foi abaixo de 5,0; analisando o baixo desempenho, observou-se que as alunas deixaram de fazer pelo menos um dos contests. As variáveis qualitativas foram obtidas através de questionário realizado com as estudantes. Entre as perguntas tem-se: i) dificuldades encontradas na disciplina Introdução à Programação, ii) se já sofreu algum preconceito, iii) se o curso contribuiu para gostar de programação, iv) se o grupo de estudos ajudou no entendimento e/ou interesse em programação, entre outros. Entre as respostas que mais chamaram a atenção, destacam-se: $66 \%$ disse já ter sofrido algum tipo de preconceito, $100 \%$ disse que começou a gostar de programação depois do curso, $100 \%$ disse que o curso ajudou na disciplina do período seguinte.

\section{Considerações finais e Trabalhos Futuros}

Este artigo apresenta um estudo de caso focal com vistas a apoiar a criação de uma proposta metodológica de ensino de programação em disciplinas de graduação. Foi programado um curso de 60 aulas, com $50 \%$ das aulas expositivas. Para o treinamento e fixação do conhecimento foi utilizado um sistema de correção automática. 75\% das alunas tiveram desempenho acima de 7,0 ao resolver as questões. Foi realizada uma pesquisa qualitativa com perguntas não estimuladas. As respostas que evidenciaram a satisfação das alunas com o curso. Elas registraram que se sentem mais motivadas em estudar programação e que o curso ajudou nas disciplinas posteriores. Como trabalhos futuros pretende-se ampliar o número de alunas, aplicar o curso em outros cenários, como por exemplo, preparatório para Olímpiada Brasileira de Programação e ampliar os conteúdos para disciplinas mais avançadas de programação.

\section{Referências}

Alves, G. M. d. S., da Silva, J. J., Dantas, R. d. Q., Dantas, V. F., de Figueiredo, R. V., $\&$ de Souza, G. C. (2017). Usando técnicas de aprendizagem colaborativa para incentivar o ensino-aprendizagem de programação entre as alunas de cursos de Computação. Paper presented at the $11^{\circ}$ Women in Information Technology (WIT 2017).

Bosse, Y., \& Gerosa, M. A. (2015). Reprovações e Trancamentos nas Disciplinas de Introdução à Programação da Universidade de São Paulo: Um Estudo 
Preliminar. Paper presented at the XXIII WEI-Workshop sobre Educação em Informática. Recife, julho. Disponível em https://drive. google. com/file/d/0BznCaSIV6ocyanVOMlhpNFh6OUk/view.

Castro, T., Fuks, H., Sposito, M. A. F., \& de Castro Júnior, A. N. J. I.-R. (2009). Análise de um Estudo de Caso para Aprendizagem de Programação em Grupo. 4(2), $155-160$.

Deters, J. I., Silva, J. d., Miranda, E., \& Fernandes, A. (2008). O desafio de trabalhar com alunos repetentes na disciplina de Algoritmos e Programação. Paper presented at the Workshop de Ambientes de apoio à Aprendizagem de Algoritmos e Programação.

Giraffa, M. M., \& da costa Mora, M. (2013). Evasão na disciplina de algoritmo e programação: um estudo a partir dos fatores intervenientes na perspectiva do aluno. Paper presented at the Congresos CLABES.

IBGE. (2010). Censo demográfico : 2010 : trabalho e rendimento : resultados da amostra. $\quad$ Retrieved 25/03, 2019, from https://biblioteca.ibge.gov.br/index.php/bibliotecacatalogo?\%20view=detalhes\& amp;id=71075

Lauschner, T., De Freitas, R., Nakamura, F., \& de Aguiar Gomes, L. (2016). Cunhantã digital: programa de incentivo a participação de mulheres da região amazônica na computação e áreas afins. Paper presented at the 10o Women in Information Technology (WIT 2016), Porto Alegre, RJ, In: Anais do XXXVI Congresso da Sociedade Brasileira de Computação (CSBC 2016).

Maciel, C., \& Bim, S. A. (2017). Programa Meninas Digitais-ações para divulgar a Computação para meninas do ensino médio. 327-336.

Pessoa, M. S. P., Tamayo, S. C., \& Franco, E. M. (2017). Panorama da Participação Feminina em Competições de Programação Promovida por Instituição de Ensino Superior da Região Norte. Paper presented at the $11^{\circ}$ Women in Information Technology (WIT 2017).

Santos, J. M. O., Pereira, K. A. d. S., \& Santos, D. A. (2018). O uso da programação para atração de mulheres à computação: relatos de experiência. Paper presented at the $12^{\circ}$ Women in Information Technology (WIT 2018).

Sousa, F. G. P., Lima, F. M. M., Bernardo, J. R. S., \& Ferreira, R. M. (2018). Criação de um Clube de Programação: uma análise da participação feminina.

Wing, J. M. (2006). Computational thinking. Communications of the ACM, 49(3), 3335.

Yin, R. K. (2015). Estudo de Caso-: Planejamento e Métodos: Bookman editora. 